\title{
Modeling the 802.11 protocol under different capture and sensing capabilities
}

\author{
Mathilde Durvy \\ EPFL \\ CH-1015 Lausanne, Switzerland \\ mathilde.durvy@epfl.ch
}

\author{
Olivier Dousse \\ Deutsch Telekom Laboratories \\ 10587 Berlin, Germany \\ olivier.dousse@telekom.de
}

\author{
Patrick Thiran \\ EPFL \\ CH-1015 Lausanne, Switzerland \\ patrick.thiran@epfl.ch
}

\begin{abstract}
Decentralized medium access control schemes for wireless networks based on CSMA/CA, such as the 802.11 protocol, are known to be unfair. In multi-hop networks, they can even favor some connections to such an extent that the others suffer from virtually complete starvation. This observation has been reported in quite a few works, but the factors causing it are still not well understood. We find that the capture effect and the relative values of the receiving and carrier sensing ranges play a crucial role in the unfairness of these protocols. We show that an idealized 802.11 protocol does suffer from starvation when the receiving and sensing ranges are equal, but quite surprisingly this unfairness is reduced or even disappears when these two ranges are sufficiently different. Using a Markovian model, we explain why apparently benign variations in these ranges have such a dramatic impact on the 802.11 protocol performance.
\end{abstract}

\section{INTRODUCTION}

The IEEE 802.11 Medium Access Control (MAC) protocol is unfair. Indeed, in single-hop networks, it has been shown that the Binary Exponential Backoff (BEB) of the 802.11 protocol creates situations where a single node has an almost exclusive access to the communication channel. In multi-hop networks, similar starvation phenomena have been reported ([1], [2], [3], [4], [5]). However, contrary to the single-hop case, very few models are able to explain the starvation problems encountered in multi-hop topologies. Existing models often concentrate on very small network topologies or describe how a specific feature of the 802.11 protocol affects its fairness. In addition, it is common to assume that the receiving and carrier sensing ranges are equal, or to neglect the the so-called "capture effect" and assume that two packets received at the same time always collide.

The goal of this work is to shed some light on how such assumptions affect the performance of the 802.11 protocol. Our approach is the following. Instead of concentrating on a specific implementation aspect of the 802.11 protocol, we consider an idealized 802.11 protocol that retains the key features of the 802.11 protocol such as its carrier sensing, collision avoidance and backoff mechanisms. Section II describes this protocol and the metrics used to evaluate its performance. We use continuous time Markov chains to describe the dynamics

The work presented in this paper was supported (in part) by the National Competence Center in Research on Mobile Information and Communication Systems (NCCR-MICS), a center supported by the Swiss National Science Foundation under grant number 5005-67322. of the protocol. We find that even though this idealized protocol does not suffer from the well-known problems that have already been identified in single-hop and multi-hop networks, it is still subject to the starvation phenomenon described in the literature. However, we also observe that the performance of the protocol are very sensitive to the assumptions made in terms of carrier sensing range and capture effect.

The main contribution of this paper is the explanation of the starvation phenomenon. In particular, we show under which condition this phenomenon occurs and why. We separate our analysis into three classes of assumptions, each leading to a different Markov chain structure. Section III covers the simplest case, that has previously been studied in the literature, and summarizes the known results. In Sections IV and $\mathrm{V}$, we develop new Markov models to address the cases where the nodes' receiving range and carrier sensing range are significantly different. These two sections differ in the underlying assumptions on the nodes' capture capability, and consequently in the structure of the Markov models involved.

\section{FRAMEWORK}

\section{A. Medium Access Control Layer}

1) Protocol Overview: In the 802.11 protocol (ad hoc mode), a node intending to transmit first senses the medium. Physical and virtual carrier sensing mechanisms are used to determine the state of the medium. The physical carrier sensing is provided by the physical layer. The virtual carrier sensing is done through an RTS/CTS handshake: The sender first sends a "request to send" packet (RTS), to which the receiver answers with a "clear to send" packet (CTS). Both packets contain information about the time at which the exchange will be completed, so that overhearing nodes refrain from emitting during the exchange.

To prevent collisions, each node maintains a backoff timer, which is initialized to a random value picked according to some backoff distribution. Timers run when the channel is idle; when a node senses the channel busy, it temporarily freezes its timer. Nodes may start emitting only when their timer reaches zero. After each transmission, the emitter resets its timer to a new random value.

2) Assumptions: In this paper, we consider an idealized version of the 802.11 protocol in order to capture its essential features, and leave aside the effects due to the imperfection of 


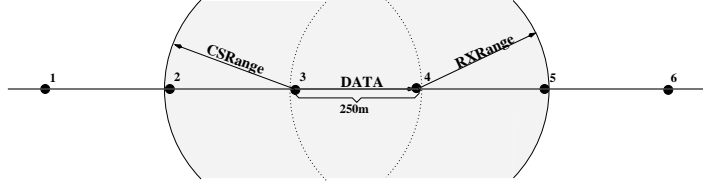

Fig. 1. CSRange $=$ RXRange $=250 \mathrm{~m}$. All nodes in the CSRange are in the RXRange, the exclusion domain is symmetric.

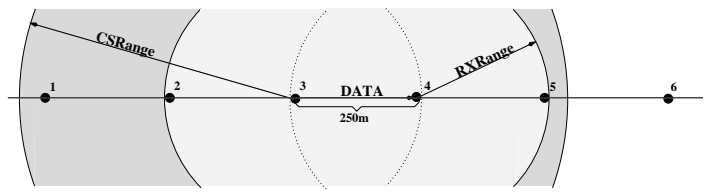

Fig. 2. RXRange $=250 \mathrm{~m}$ and CSRange $=550 \mathrm{~m}$. As the CSRange is larger than the RXRange, more nodes are silenced around the sender (Node 3). The exclusion domain is asymmetric.

the real protocol (we refer the reader to [6] for an overview of those effects). First, we assume that nodes can accurately detect the activity on the communication channel by using their physical and virtual carrier sensing mechanisms. In addition, we consider a continuous backoff distribution, instead of the discrete distribution implemented in actual 802.11 cards, so that two timers cannot expire at the same time. These two assumptions imply that the idealized protocol is collision free. We also use a separate backoff timer for each link, even if they share the same source node, as recommended by [7] in an attempt to guarantee per link fairness at least in the context of single-hop networks. Unless otherwise specified, we consider exponentially distributed backoff and exchange times. Our analysis assumes saturated traffic conditions.

3) Interaction with the Physical Layer: We model the physical layer using two parameters: the receiving range and the carrier sensing range.

The receiving range (RXRange) is the maximum distance from the source at which a packet can be successfully received in the absence of interfering nodes. The carrier sensing range (CSRange) is the maximum distance from the emitter at which a transmission can still be detected. The CSRange is always larger than the RXRange. All packets received from nodes within CSRange are forwarded to its MAC layer. However, only packets received from nodes within RXRange can be successfully processed.

After the initial RTS-CTS handshake, nodes in the RXRanges of the sender and of the destination are silenced by their virtual carrier sensing mechanism. In addition, nodes within CSRange of the sender are kept from sending by their physical carrier sensing mechanism. We refer to the domain silenced around an active link by its virtual and physical carrier sensing as its exclusion domain. In this work we distinguish between symmetric exclusion domains (Figure 1) and asymmetric exclusion domains (Figure 2).

\section{B. Metrics}

We use two metrics to characterize the performance of the 802.11 protocol:
1) Spatial Reuse $(\sigma)$ : We define the average spatial reuse as the average fraction of active links (over time) in the network.

2) Fairness Index (FI): To assess the MAC layer fairness of the protocol we use Jain's Fairness Index. Consider a network with $L$ links and denote by $p(j)$ the probability that the $j^{\text {th }}$ link be active under a given medium access control protocol. The link fairness index of the protocol is FI $=\frac{\left(\sum_{j} p(j)\right)^{2}}{L \sum_{j} p(j)^{2}}$. The maximum fairness index is 1 . It corresponds to a network where all links access the channel equally. Yet, if only $k$ links have an equal access to the channel and the remaining links have no access to the channel, the fairness index is $k / L$.

\section{ThE SYMMETRIC CASE}

\section{A. Specific Assumptions}

In this section, we assume that all exclusion domains are symmetric. The exclusion domain around a link is symmetric if no node is located outside the RXRange of the emitter while inside its CSRange (see example in Figure 1). We call the domain symmetric because the reverse link has the same exclusion domain. In this setting, transmissions never interfere with each other, as all nodes in the receivers' CSRange are properly silenced by the CTS packets. The case with symmetric exclusion domain has already been studied in [1] and [2]. We summarize known results in this section.

\section{B. Markov Modeling}

The dynamics of our idealized 802.11 protocol can be described by a continuous Markov chain. At any given time, a set of directional links is active in the network. Such a set is called a transmission pattern and defines a state of the Markov chain. Clearly, only patterns that respect the constraints set by the exclusion domains of active links are possible. Hence the set of all valid patterns forms the state space of the Markov chain. We say that a state is at level $i$ if the corresponding transmission pattern has exactly $i$ active links, and we denote by $N(i)$ the number of such states. The states at the highest level are called patterns of maximal spatial reuse.

Transitions in the Markov chain can only occur between states that are separated by one level: A transition between a state at level $i$ and a state at level $i-1$ (respectively at level $i+1$ ) corresponds to the completion (resp. beginning) of a transmission.

Denote by $\mu^{-1}$ the average exchange time and by $\lambda^{-1}$ the average backoff time. The transition rate between a state at level $i$ and a state at level $i-1$ (respectively at level $i+1$ ) is $\mu$ (resp. $\lambda$ ). Figure 3 gives an example of such a Markov chain. Finally, we define the access intensity as $\rho:=\lambda / \mu$.

The stationary probability of any state at level $i$ is equal to

$$
\pi(i)=\frac{\rho^{i}}{\sum_{k} N(k) \rho^{k}} .
$$

The average spatial reuse is then $\sigma=\frac{1}{L} \sum_{i} i N(i) \pi(i)$. To derive the long-term fairness of the protocol, we need to compute, in addition, the probability that a specific link is active. Denote by $N(i, j)$ the number of transmission patterns 


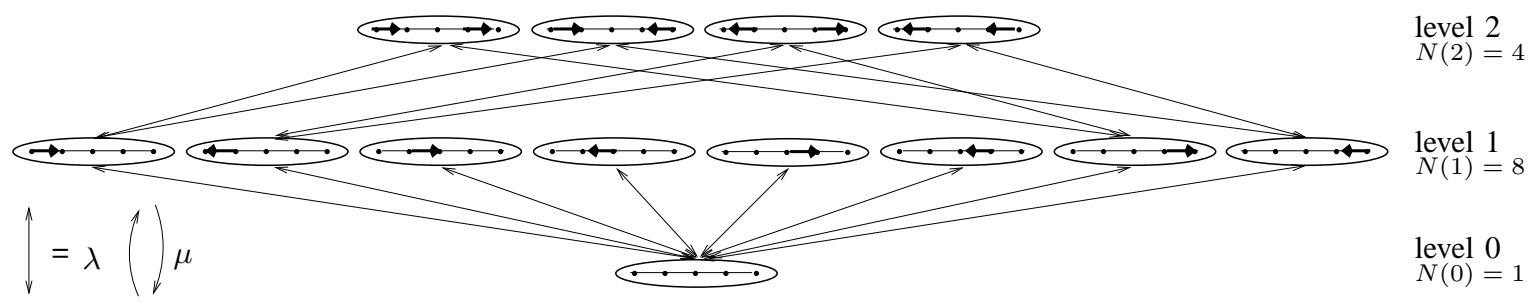

Fig. 3. Markov chain for a small line topology of 5 nodes and with the RXRange and the CSRange covering exactly one neighbor.

$\begin{array}{lllllllllllllllll}0 & 1 & 2 & 3 & 4 & 5 & 6 & 7 & 8 & 9 & 10 & 11 & 12 & 13 & 14 & L-1 & L\end{array}$ 1 space unit $\rightarrow$ interval of length $l=3$

Fig. 4. A transmission pattern of maximal spatial reuse with symmetric exclusion domains (both RXRange and CSRange covering one neighbor).

that contain link $j$ at level $i$. The probability $p(j)$ that link $j$ is active is $p(j)=\sum_{i} N(i, j) \pi(i)$, and the fairness index can be computed using the definition of Section II-B.2.

For $\rho>1, \pi(i)$ increases with the value of $i$, and the transmission patterns with a high number of active links have an increased probability of appearing, compared to those with only a few active links. In the limit $\rho \rightarrow \infty$, only the patterns of maximizal spatial reuse have a non-trivial stationary probability. Consequently, when the average backoff time is much lower than the average exchange time, the idealized 802.11 protocol achieves the maximal spatial reuse. However, all links that do not belong to the transmission patterns of maximal spatial reuse are completely starved.

The Line Topology: It is in general not possible to obtain a closed form expression for $N(i)$ nor $N(i, j)$. However, we can do so for a line topology, where $L+1$ nodes (numbered from 0 to $L$ ) are equally spaced (by 1 space unit) along a straight line (Figure 4).

We define the parameter $l$ as 1 plus the minimal distance separating two non-colliding active transmissions. This means that there can be an active transmission every $l$ space units (Figure 4). Therefore, finding possible transmission patterns boils down to packing intervals of length $l$ in a line segment of length $L+(l-1)$ (the additional term $(l-1)$ compensates for the border effects). The number of valid transmission patterns with $i$ active links is thus

$$
N(i)=2^{i}\left(\begin{array}{c}
i+v \\
i
\end{array}\right)
$$

where $v=L+(l-1)-l i$ is the length of the vacant space on the line once $i$ intervals of length $l$ are placed. Similarly,

$$
N(i, j)=2^{i} \sum_{k=0}^{i-1}\left(\begin{array}{c}
k+v_{\text {left }} \\
k
\end{array}\right)\left(\begin{array}{c}
(i-1)-k+v_{\text {right }} \\
(i-1)-k
\end{array}\right),
$$

where $v_{\text {left }}=j-1-l k$ and $v_{\text {right }}=L-j-l((i-1)-k)$. Using the expressions for $N(i)$ and $N(i, j)$ we can compute analytically the spatial reuse and the fairness index of the protocol on a finite line topology. Note that these values depend on the total length on the network $L$. For large networks, i.e. when $L \rightarrow \infty$, the spatial reuse is shown in [2] to converge to

$$
\sigma(\rho) \sim \frac{2 \rho y_{1}^{l-1}}{1+2 l \rho y_{1}^{l-1}}
$$

where $y_{1}$ is the real root of $1-y-2 \rho y^{l}$ closest to the origin.

\section{The Asymmetric Case with Full Capture EFFECT}

\section{A. Specific Assumptions}

The exclusion domain around a link is asymmetric if there are nodes inside the emitter's CSRange that are outside its RXRange (as Node 1 in Figure 2). These nodes are unable to decode the RTS packets from the emitter and are silenced only through the physical carrier sensing. Therefore, more nodes are silenced on the emitter's side, and this is why we call the exclusion domain asymmetric.

Now assume that a node in the receiver's CSRange (for example Node 6 in Figure 2) begins transmitting. As the two nodes are within CSRange of each other, the new transmission interferes with the ongoing transmission. We assume that the signal-to-interference ratio is still high enough for the receiver to keep decoding its packet, despite the new interferer (capture effect).

Furthermore, we assume that the capture effect also occurs when the stronger signal begins after the weaker one. This implies in particular that in the above scenario, the two transmissions can also be established in the reverse order. Full capture is a rather optimistic scenario, although it is supported to some extent by experimental evidences [8].

\section{B. Simulation Results}

Figures 5 and 6 show that in the asymmetric case the spatial reuse of the 802.11 protocol increases less rapidly and that we are able to maintain a slightly better fairness than in the symmetric case. The comparison of Figures 7(b) and 7(a) confirms that the starvation phenomenon is less pronounced than in the symmetric case and that the links in the middle of the line topology get a fairer access to the channel. Although these results are quite comforting and definitively show that, for a given access intensity $\rho$, the protocol can have different behaviors, we will see in the analysis that follows that the spatial reuse and the fairness index of the 802.11 protocol in the asymmetric case with full capture actually converge as $\rho \rightarrow \infty$ to the same values as in the symmetric case. 


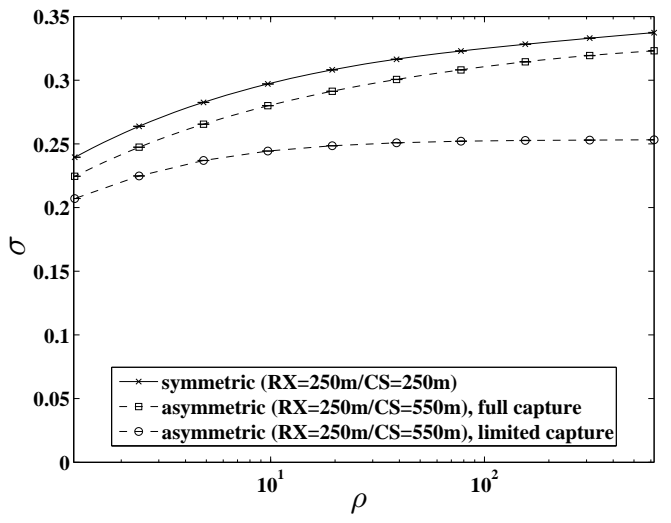

Fig. 5. Spatial reuse achieved by the idealized 802.11 protocol on a line topology of 50 nodes, as a function of the access intensity $\rho$. The markers correspond to the results obtained by simulations (code available at [9]) and the curve to the results obtained analytically (except for the 'asymmetric limited capture' case where we do not have the exact analytical curve).

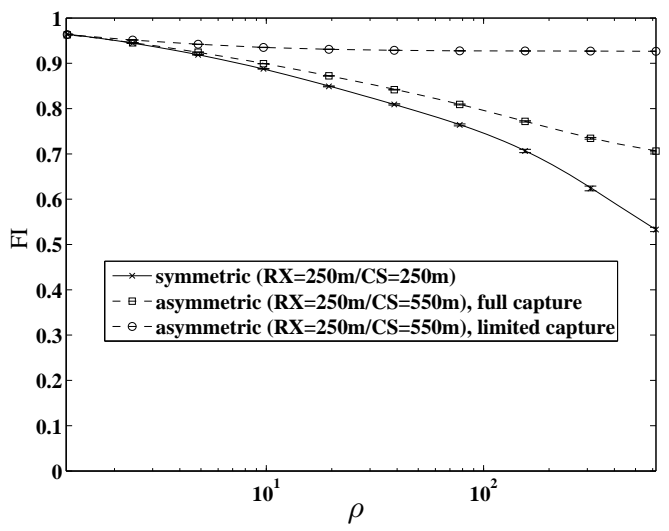

Fig. 6. Fairness index of the idealized 802.11 protocol on a line topology of 50 nodes.

\section{Markov Modeling}

The structure of the Markov chain remains essentially unchanged but some states (and the corresponding transitions) disappear. Take for example the Markov chain of Figure 3 and assume that the CSRange now covers two neighbors instead of one. All transmission patterns remain valid except the third pattern at the top level of the Markov chain, where two transmitters are back to back. This change in the set of valid transmission patterns does not affect the reversibility of the Markov chain. Equation (1) still holds, and the asymptotic properties of the protocol for $\rho \rightarrow \infty$ are thus unchanged. However, the number $N(i)$ of valid transmission patterns with $i$ active links and the number $N(i, j)$ of such patterns including link $j$ have to be recomputed.

The Line Topology: We start with the observation that two consecutive active links along the line are separated by the least number of space units if their orientation is such that the two senders are not back to back. Consider a transmission pattern of maximal spatial reuse and assume that, once we have placed the intervals corresponding to its active links, there is no vacant space; because of the above observation, all active links must point in the same direction until a certain point,

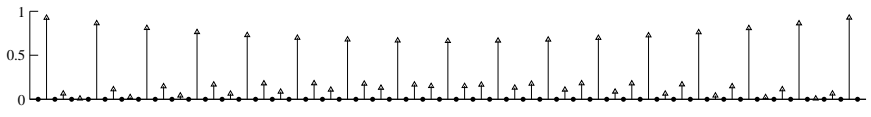

(a) Symmetric exclusion domains (RXRange $=$ CSRange $=250 \mathrm{~m}$ ). $\sigma=0.34$, FI $=0.53$.

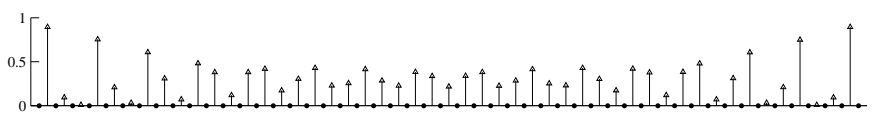

(b) Asymmetric exclusion domains (RXRange $=250 \mathrm{~m}$, CSRange $=550 \mathrm{~m}$ ) with full capture. $\sigma=0.32, \mathrm{FI}=0.70$.

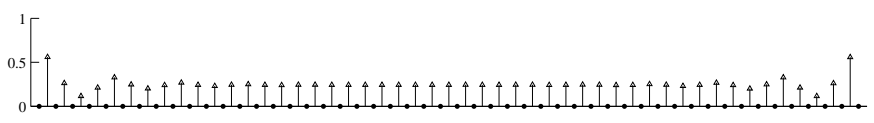

(c) Asymmetric exclusion domains (RXRange $=250 \mathrm{~m}$, CSRange $=550 \mathrm{~m}$ ) with limited capture. $\sigma=0.25, \mathrm{FI}=0.93$.

Fig. 7. On the $x$ axis, a line topology of 50 nodes. To each link $j$ we associate a vertical bar that corresponds to the probability $p(j)$ that the link be active in the simulation for $\rho=620$.

and then point to the other direction (see Figure 8). If $i$ is the number of active transmissions, there are clearly $i+1$ possible settings. Now, let us assume that there is one vacant

\section{Direction change}

Fig. 8. A transmission pattern of maximal spatial reuse $(l=3)$. Active links are sorted in two groups, inside which all links point in the same direction.

space. This vacant space will allow for an additional change of direction, as depicted in Figure 9. Therefore, in this case,

$$
\text { Vacant space }
$$

Fig. 9. A transmission pattern with one vacant space $(l=3)$. There are three points where links change direction.

there is a total of three points where links change direction and there are $\left(\begin{array}{c}i+3 \\ i\end{array}\right)$ ways to pick these three points. This reasoning can be extended to an arbitrary number of vacant spaces $v$, and we find

$$
N(i)=\left(\begin{array}{c}
i+2 v+1 \\
i
\end{array}\right)
$$

where $v=L+(l-1)-l i$.

Denote by $j_{R}$ the link from node $j-1$ to node $j$, we have

$$
N\left(i, j_{R}\right)=\sum_{k=0}^{i-1}\left(\begin{array}{c}
k+2 v_{\text {left }} \\
k
\end{array}\right)\left(\begin{array}{c}
(i-1)-k+2 v_{\text {right }}+1 \\
(i-1)-k
\end{array}\right)
$$

where $v_{\text {left }}=j-1-l k$ and $v_{\text {right }}=L-j-l((i-1)-k)$. By doing the same for the link in the opposite direction, namely from node $j$ to node $j-1$, we get $N(i, j)=$ $N\left(i, j_{R}\right)+N\left(i, j_{L}\right)$. We can then plug the expressions for $N(i)$ and $N(i, j)$ in the equations of Section III-B to obtain the exact curve for the spatial reuse and the fairness index of the protocol. 


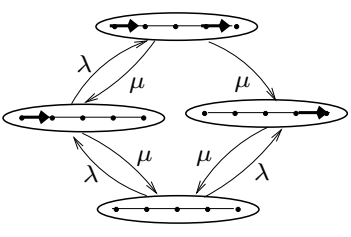

Fig. 10. A small part of the non-reversible Markov chain.

For $L \rightarrow \infty$ we obtain

$$
\sigma(\rho) \sim \frac{2 \rho y_{1}^{2 l-1}}{1+2 l \rho y_{1}^{2 l-1}}
$$

where $y_{1}$ is the real root of $1-y-\rho y^{2 l}$ closest to the origin. Again, we verify that this expression tends indeed to $1 / l$ when $\rho$ tends to infinity.

\section{The Asymmetric Case with Limited Capture} EFFECT

\section{A. Specific Assumptions}

Limited capture means that the capture effect occurs only if the strongest signal comes first. This assumption reflects the fact that in practice, many radio circuits cannot resynchronize to a stronger signal if they are already locked on another (weaker) carrier. Consequently, in this capture model, the order of arrival of the transmissions does matter. This limited capture effect is implemented in the most well-known network simulators, including Ns-2 and Qualnet.

\section{B. Simulation Results}

These simulation results were at first so surprising to us that they triggered the work presented in this paper. Figures 5 and 6 show the performance of the idealized 802.11 protocol with asymmetric exclusion domains and limited capture. Its spatial reuse increases to a value much below $1 / 3$ but its fairness index remains above 0.9 , even for large values of $\rho$. The access probabilities of the different links presented in Figure 7(c) illustrate even better the dramatic improvement of the protocol fairness at high access intensity. Except at the borders, the situation is now completely fair.

\section{Markov Modeling}

The set of the valid transmission patterns is unchanged from the case with full capture effect. However, some states can now be reached only from a restricted set of lower states. Figure 10 illustrates a small part of the Markov chain used to model the 802.11 protocol with limited capture effect. We see that, compared to the original Markov chain, a directed edge has been removed: it accounts for the fact that the state at the top level can only be reached if the links become active in the right order. Removing possible transitions between the states of the Markov chain breaks its regular structure, and the chain looses its reversibility property. This makes the analytical study of the chain very difficult as its stationary distribution does not have a nice product form anymore. However, a careful observation of the structure of the chain allows us to explain the results observed by simulation.
Let us first look back at the reversible case (corresponding to the two previous sections). For large values of $\rho$, the chain spends most of its time in the states of maximal spatial reuse. Indeed, when a transition occurs from a state at the top level to a state at the lower level (i.e., when an active link becomes idle), as $\lambda \gg \mu$, the next transition is most likely to be the reactivation of the same link, bringing the chain back to the same top level state. In the non-reversible case, this cannot happen anymore, because many of the transitions from top level states to lower level states cannot be reverted. Therefore, if the chain leaves a top level state, it might have to go down two or more levels before it can climb back to a top level state. This new dynamic has two consequences. After leaving a top level state, the chain has a non-negligible probability to return to a different top level state. This implies that a different set of links will be active, thus restoring fairness. Second, the time spent in non-maximal spatial reuse states (states below the top level) is also non-negligible. As a consequence, in contrast to the previous cases, the average spatial reuse does not tend to the optimal value for increasing $\rho$ but the fairness index remains strictly larger than $1 / l$.

\section{CONCLUSION}

Using continuous Markov chain models, we explained the unfairness of the 802.11 protocol in multi-hop ad hoc networks, but also showed that its performance varies with its capture and sensing capabilities. We found that for a given (finite) access intensity $\rho$, the 802.11 protocol is fairer with asymmetric than with symmetric exclusion domains. In addition, we demonstrated that the capture capabilities of the protocol play a decisive role on its performance when $\rho \rightarrow \infty$. In the asymmetric case with full capture, the spatial reuse of the 802.11 protocol is maximal, but all the links that do not belong to the patterns of maximal spatial reuse are starved. In contrast, in the asymmetric case with limited capture, the spatial reuse of the protocol is not maximal but starvation can be avoided.

\section{REFERENCES}

[1] X. Wang and K. Kar, "Throughput Modelling and Fairness Issues in CSMA/CA Based Ad-Hoc Networks," in INFOCOM, Miami, 2005.

[2] M. Durvy and P. Thiran, "A Packing Approach to Compare Slotted and Non-Slotted Medium Access Control," in INFOCOM, Barcelona, 2006.

[3] M. M. Carvalho and J. J. Garcia-Luna-Aceves, "A scalable model for channel access protocols in multihop ad hoc networks," in MobiCom. ACM Press, 2004, pp. 330-344.

[4] M. Garetto, T. Salonidis, and E. Knightly, "Modeling Per-flow Throughput And Capturing Starvation in CSMA Multi-hop Wireless Networks," in INFOCOM, Barcelona, 2006.

[5] K. Medepalli and F. Tobagi, "Towards Performance Modeling of IEEE 802.11 based Wireless Networks: A Unified Framework and its Applications," in INFOCOM, Barcelona, 2006.

[6] M. Durvy and P. Thiran, "Understanding the Gap between the IEEE 802.11 Protocol Performance and the Theoretical Limits," in SECON, Reston, 2006.

[7] V. Bharghavan, A. Demers, S. Shenker, and L. Zhang, "MACAW: a media access protocol for wireless LAN's," in SIGCOMM. ACM Press, 1994, pp. 212-225.

[8] A. Kochut, A. Vasan, A. Shankar, and A. Agrawala, "Sniffing out the correct physical layer capture model in 802.11 b," in ICNP, 2004

[9] [Online]. Available: http://icapeople.epfl.ch/mdurvy/research.html 